\title{
Performance analysis of MSE-OFDM system
}

\author{
Poonam Singh ${ }^{1}$, Saswat Chakrabarti ${ }^{2}$ \\ ${ }^{1}$ Electronics \& Comm. Engg. Dept. National Institute of Technology Rourkela, India \\ ${ }^{2}$ GSSST, Indian Institute of Technology, Kharagpur, India
}

\section{Email address:}

psingh@nitrkl.ac.in(P.Singh), saswat@ece.iitkgp.ernet.in(S. Chakrabarti)

\section{To cite this article:}

Poonam Singh, Saswat Chakrabarti. Performance Analysis of MSE-OFDM System. International Journal of Wireless Communications and Mobile Computing. Vol. 1, No. 1, 2013, pp. 41-50. doi: 10.11648/j.wcmc.20130101.17

\begin{abstract}
In this paper we analyze the effects of interchannel interference (ICI) and intersymbol interference (ISI) on the performance of a Multi-Symbol Encapsulated Orthogonal Frequency Division Multiplexing (MSE-OFDM) system. MSEOFDM is a bandwidth efficient OFDM scheme, where a number of OFDM symbols are grouped together as a frame and protected by one single cyclic prefix. This reduces the extent of redundancy caused by the CP and increases the bandwidth efficiency of the system. We have derived expressions for probability of error for MSE-OFDM in presence of ICI and ISI, which result from the time variation and delay spread of mobile channels. Both analysis and simulation results are presented for the MSE-OFDM system and are found to be almost identical.
\end{abstract}

Keywords: OFDM, MSE-OFDM, Bandwidth Efficiency, Synchronization Errors, PAPR

\section{Introduction}

Orthogonal Frequency Division Multiplexing (OFDM) has been applied widely in wireless communication systems due to its high data rate transmission capability with high bandwidth efficiency and its robustness to multipath delay. Another advantage of OFDM is its simple receiver structure using a frequency domain equalizer with only one complex multiplication per subcarrier. This is achieved by introducing a cyclic prefix, which is a cyclic extension of the output sequence, to eliminate intersymbol interference among the symbols [1]. Therefore, an appreciable amount of redundancy is introduced in an OFDM system which reduces the bandwidth efficiency. To avoid this redundancy, an OFDM system called MultiSymbol Encapsulated Orthogonal Frequency Division Multiplexing (MSE-OFDM) has been proposed in literature [2-6]. OFDM is also sensitive to frequency and timing synchronization errors. The peak-to-average power ratio (PAPR) is also high, which limits the efficiency of the power amplifier. The effects of these phenomena can be reduced by using MSE-OFDM.

Two different implementations of the MSE-OFDM scheme have been proposed [2] [5]. The first implementation, termed the CP-reduced system, is designed to improve the bandwidth efficiency for static channels by reducing the number of $\mathrm{CP}$ insertions. The bandwidth efficiency is improved as the MSE-OFDM frame size increases. The other implementation, termed FFT-size reduced system, is designed to keep the MSEOFDM frame duration same as that of a conventional OFDM symbol i.e. the symbol duration reduces for the MSE-OFDM system, while the bandwidth efficiency remains the same. This reduces the number of subcarriers and the FFT size of MSE-OFDM system. So the PAPR and robustness to frequency offset improves.

An accurate channel estimation is required for the receiver design. Channel estimation for OFDM is usually performed in the frequency domain by either inserting pilot tones into all subcarriers of the OFDM symbols with a specific period or by inserting some pilot tones into each OFDM symbol. Several pilot-aided channel estimation schemes have been investigated for OFDM applications. Similar channel estimation methods can be used for MSEOFDM systems.

Similar to conventional OFDM systems, MSE-OFDM are also very sensitive to frequency offsets due to the loss of orthogonality among the subcarriers. Various techniques have been proposed for frequency offset estimation and correction of OFDM systems [7-8]. Timing acquisition detects the presence of a new frame in the received data stream and once the frame is detected, it provides a coarse estimate of the timing error to find the correct position of the received DFT window. A popular timing acquisition 
algorithm was proposed by Schmidl and Cox [9]. A joint estimation of the frequency offset and the channel response were investigated [10] to improve the system performance and minimize the estimation errors. Similar channel estimation and frequency and timing synchronization schemes can be implemented for MSE-OFDM systems. There may be residual frequency and timing offsets even after synchronization techniques are used. The intercarrier interference (ICI) due to residual frequency offset will affect the accuracy of the OFDM channel estimation. We have analyzed and simulated the effects of these residual offsets on MSE-OFDM systems.

In this chapter, we analyze the performance of MSEOFDM systems in AWGN and frequency selective Multipath Fading Channels. Channel variations severely degrade the performance of MSE-OFDM by introducing both a complicated multiplicative distortion and an additive intercarrier interference. The effects of carrier frequency offset and timing offset on MSE-OFDM are studied. We have done extensive simulations for these cases and verified the results using analysis. Section 2 gives a general description of the MSE-OFDM system. In Section 3 we have derived expressions for probability of error in presence of synchronization errors in multipath fading channels. Simulation results are given in Section 4 and some conclusions are given in Section 5.

\section{The MSE-OFDM System Description}

MSE-OFDM is a bandwidth efficient scheme, where a number of OFDM symbols are grouped together and a single cyclic prefix is used [5]. In CP-reduced MSEOFDM, a number of OFDM symbols are grouped together in a frame and a single cyclic prefix is used in one frame. This reduces the redundancy caused by cyclic prefix in each symbol and therefore improves the bandwidth efficiency. In FFT-reduced MSE-OFDM system, each OFDM symbol is divided into smaller IFFT blocks, which are protected by one single cyclic prefix. The number of subcarriers is reduced, which reduces the effects of frequency offset and also reduces the PAPR. The bandwidth efficiency is same as that of OFDM.

The block diagram for the transmitter of the MSEOFDM system is shown in Fig.1, where $\mathrm{N}$ and $\mathrm{M}$ denote the size of IFFT modulator and the total number of OFDM symbols in one MSE-OFDM frame respectively.

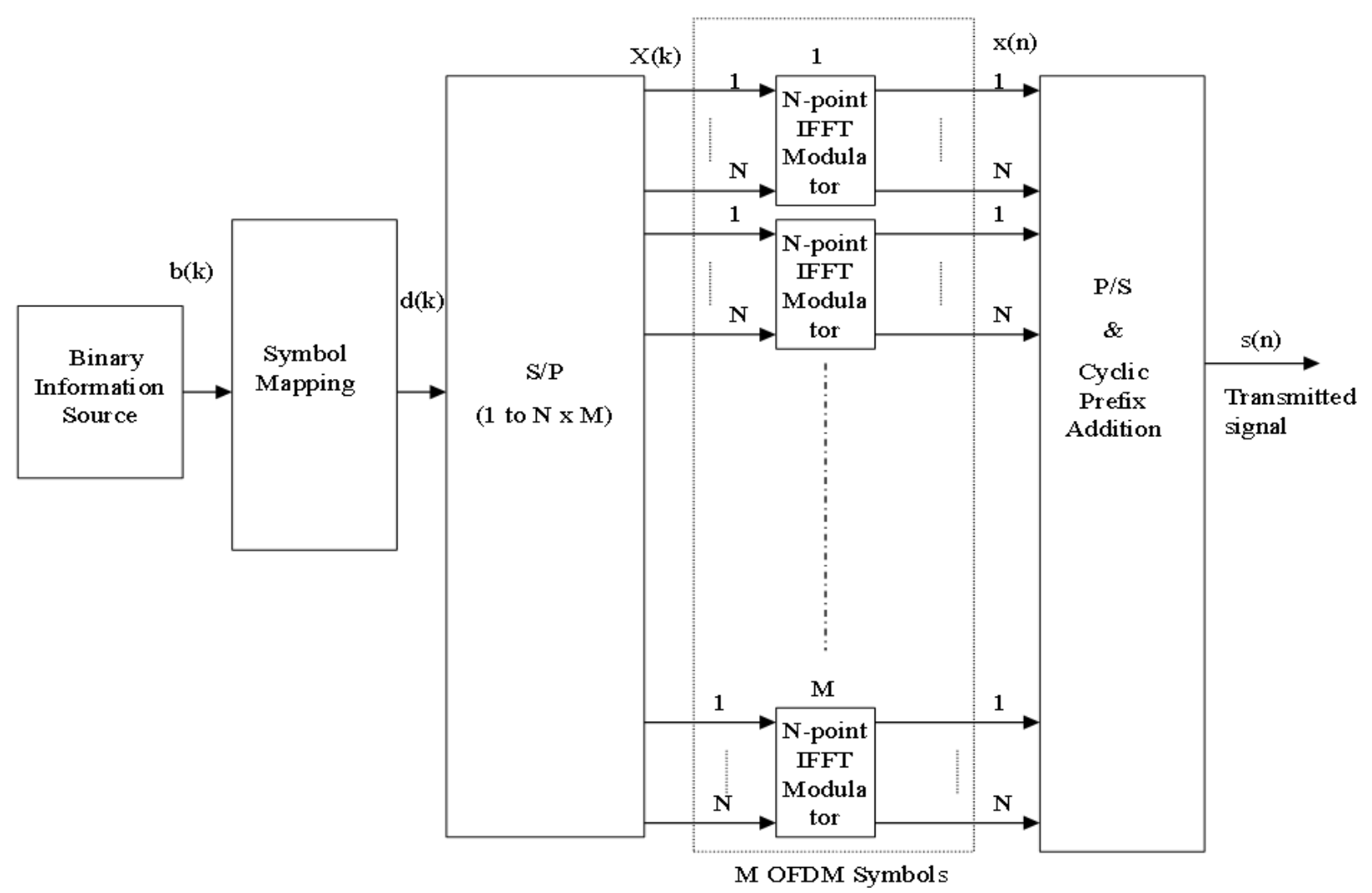

Fig 1. Transmitter of MSE-OFDM, N: Number of data samples used to generate one OFDM symbol, M: Number of OFDM symbols in one MSE-OFDM frame, $N_{c p}$ : number of samples used as cyclic prefix.

To generate one frame of MSE-OFDM signal, M OFDM symbols are generated and then a cyclic extension of Ncp samples of last OFDM symbol in the same frame is inserted as the cyclic prefix. Here, one cyclic prefix is used for one frame which consists of M OFDM symbols. The OFDM signal consists of $\mathrm{N}$ complex exponentials or subcarriers which have been modulated with the complex input data $\mathrm{X}(\mathrm{k})$. Each OFDM symbol is generated by 
taking the $\mathrm{N}$ point complex modulation sequence through IDFT.

The subcarriers of the transmitted signal pass through frequency non-selective Rayleigh fading channel, and are also subjected to additive white Gaussian noise (AWGN) $n(t)$, with a double-sided power spectral density No/2. The statistics of all the Rayleigh fading channels are assumed to be identical. Furthermore, we assume that the channel fading is slow and remains unchanged over two consecutive symbols. The complex impulse response of the
Rayleigh fading channel for the m-th subcarrier can be written as

$$
h_{m}(t)=\beta_{m} e^{j \theta_{m}} \delta(t)
$$

where $\theta \mathrm{m}$ is a random phase introduced by the channel, it is modeled as uniformly distributed over the interval of $[0$, $2 \pi]$ and is assumed to be i.i.d. for each subcarrier and each user, $\beta \mathrm{m}$ is a Rayleigh random variable with power $E[\beta 2 \mathrm{~m}]=\sigma 2$ where $E[$.$] is the expectation operator.$

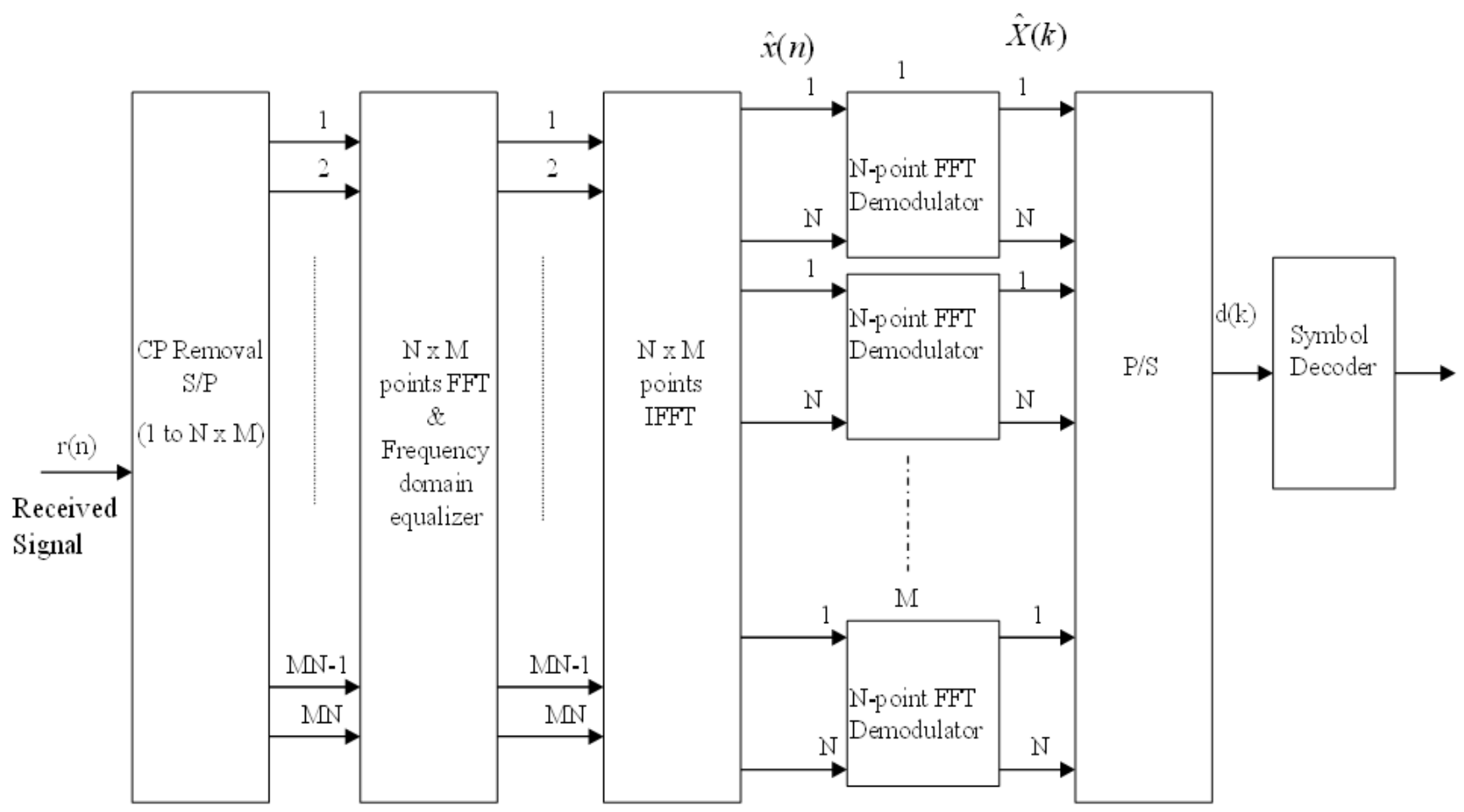

Fig 2. MSE-OFDM Receiver

\subsection{Receiver}

The MSE-OFDM receiver is shown in Fig.2. In OFDM receiver, after removing the cyclic prefix and taking inverse OFDM the received sequence is equalized to get the transmitted data. After channel estimation, the output of each subcarrier is equalized. Finally, the regenerated symbol sequences are parallel to serial converted to recover the transmitted binary data. With the help of cyclic prefix, simple frequency domain equalizer can be realized for the OFDM system. However, a new frequency domain equalizer has been employed due to the unique frame structure of the MSE-OFDM signal as shown in MSEOFDM receiver in Fig.2 [2] [5]. Channel estimation is used to compensate for the amplitude and phase distortions associated with the received signal. To estimate the multiplicative channel response, pilot symbols are inserted among the transmitted data symbols. The receiver estimates the channel state information based on the received, known pilot symbols. $\mathrm{MN}$ point FFT is performed to convert the whole frame to frequency domain. After channel estimation, one tap equalizer is used to compensate the channel distortions. For demodulation of each OFDM symbol in the same frame, the equalized frequency domain signal is converted back to time domain for IFFT demodulation. The equalized signal $\tilde{r}_{l}^{F E Q}$ is then split into M OFDM symbols for demodulation using Npoint FFT.

To implement this frequency domain equalizer, a very large MN size Fast Fourier Transformation is needed, which increases the computational complexity of the system. However, the complexity can be reduced by using a time domain ISI cancellation process as proposed in [11]. In this case, the frequency domain equalization can be done on each individual OFDM symbols using only N point FFT.

\section{Performance Analysis of MSE-OFDM}

The performance analysis of OFDM signal has been carried out in several papers with and without 
synchronization errors [12] [13]. We have extended this work for MSE-OFDM schemes. The analysis of MSEOFDM signal is similar to OFDM if there is no interference among the OFDM symbols in a frame. However, an ISI will occur among the OFDM symbols within the same MSE-OFDM frame prior to equalization. The accuracy of channel estimation is affected if the ISI is not suppressed. To reduce this ISI, a new frequency domain equalizer has been used due to the unique frame structure of the MSE-OFDM signal as explained in Section 1. In the receiver of MSE-OFDM systems, NM point FFT is performed to convert the whole frame to frequency domain. After channel estimation, one tap equalizer is used to compensate the channel distortions. For demodulation of each OFDM symbol in the same frame, the equalized frequency domain signal is converted back to time domain for IFFT demodulation. The equalized signal $\tilde{r}_{l}^{F E Q}$ is then split into M OFDM symbols for demodulation using $\mathrm{N}$ point FFT.

In practical situations, Doppler shifts and oscillator instabilities result in a carrier frequency offset between the received carrier and the local sinusoids used for signal demodulation. A carrier frequency offset produces a shift of the received signal in the frequency domain and may result in a loss of mutual orthogonality among subcarriers. This causes ICI, which may cause severe performance degradation and so must be properly compensated.

In multicarrier systems, the DFT window should include samples from only one single block in order to avoid inter symbol interference (ISI). A timing offset in this DFT window may cause ISI among the symbols. If the length of the cyclic prefix (CP) is selected to be greater than the channel impulse response (CIR) duration, no ISI is present in the DFT output and it only results in a cyclic shift of the received OFDMA block. Thus the timing error $\Delta \theta$ appears as a linear phase across subcarriers and it can be compensated for by the channel equalizer, which cannot distinguish between phase shifts introduced by the channel and those caused by timing misalignments. We have analyzed the effects of carrier frequency offset and timing offset on the performance of MSE-OFDM.

Mathematical models have been used to find the analytical solutions for the problems. First, the analysis has been done for the ideal case, where we assume that there is only AWGN noise in the channel and there is no multipath fading or interference of any kind. Then the analysis is carried out for a multipath fading channel assuming no interferences. Then the effects of carrier frequency offset and timing offset in a multipath fading channel has been considered for the analysis. All the schemes have been analyzed and also been simulated and interpreted extensively.

The transmitter and receiver models for analysis of the MSE-OFDM system are shown in Fig.3 and Fig.4. Here, one cyclic prefix is used for one frame which consists of $M$ OFDM symbols. The cyclic prefix is the cyclic extension of Ncp samples of the last OFDM symbol in the same frame. The IFFT size of modulator is $\mathrm{N}$ and the length of cyclic prefix is Ncp samples.

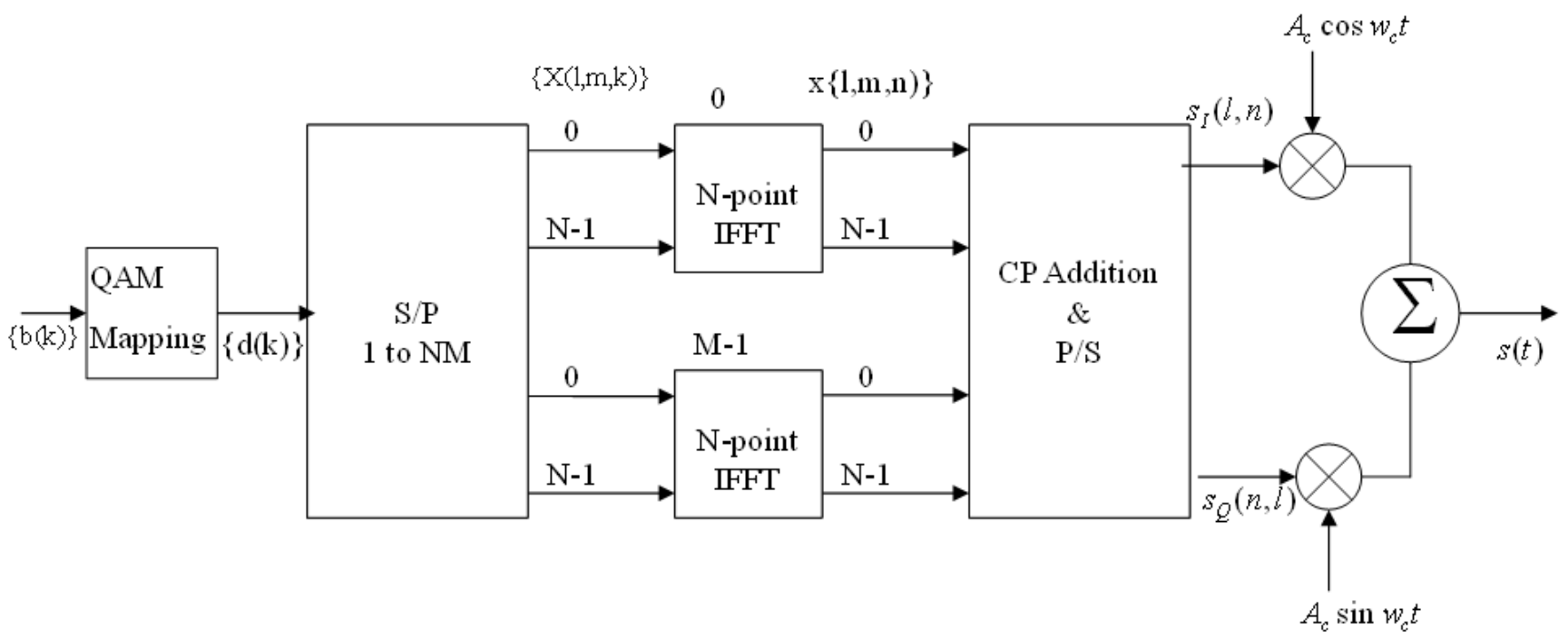

Fig 3. Transmitter of MSE-OFDM indicating major signal processing blocks

The serial binary data sequence $\{\mathrm{b}(\mathrm{k})\}, 0 \leq k<a N M$, is the input data $b(k)= \pm 1$.

This is QAM modulated to get the sample sequence $\{\mathrm{d}(\mathrm{k})\}, 0 \leq k<N M$,

$$
d(k)=d_{I}(k)+j d_{Q}(k), 0 \leq k<N M
$$

where $d_{I}(k)$ and $d_{Q}(k)$ can have a value of either \pm 1 .
After serial to parallel conversion, a complex sequence $\{X(m, k)\}$ of $\mathrm{N}$ samples of $\mathrm{d}(\mathrm{k}), 0 \leq k<N$, form one set of input to the $\mathrm{m}$-th IFFT block which generates the $\mathrm{m}$-th OFDM sample set $\{x(m, n)\}, 0 \leq n<N$. Each OFDM sample is given by

$$
x(m, n)=\sum_{k=0}^{N-1} X(m, k) \exp (j 2 \pi n k / N), \begin{aligned}
& 0 \leq n<N \\
& 0 \leq m<M
\end{aligned}
$$


In MSE-OFDM, M OFDM symbols, each having $\mathrm{N}$ samples, are taken in one frame and $\mathrm{N}_{\mathrm{cp}}$ samples from the last OFDM sample are used as cyclic prefix. Thus one frame consists of $\mathrm{NM}+\mathrm{N}_{\mathrm{cp}}$ samples.

The n-th sample of MSE-OFDM signal can now be written as

$$
\begin{aligned}
& s(n)=\sum_{k=0}^{N-1} X(M-1, k) \exp \left(j 2 \pi k\left(N-N_{c p}+n\right) / N\right), 0 \leq n<N_{c p} \\
& +\sum_{m=0}^{M-1} \sum_{k=0}^{N-1} X(m, k) \exp \left(j 2 \pi k\left(n-N_{c p}-m N\right) / N\right), N_{c p} \leq n<N M+N_{c p}
\end{aligned}
$$

where the first term represents the cyclic prefix and the second term represents the actual data to be transmitted.

As shown in Fig.4, the received signal $r(t)$ is demodulated to baseband frequency using carriers recovered from the received signal, $\cos \left[\mathrm{w}_{\mathrm{c}} \mathrm{t}+\theta(\mathrm{t})\right]$ and $\sin \left[\mathrm{w}_{\mathrm{c}} \mathrm{t}+\theta(\mathrm{t})\right]$. The double frequency term is removed by using a filter and filtered signal is integrated and sampled at an interval of $\mathrm{T}_{\mathrm{s}}+\tau$ to get the in-phase and quadrature components $r_{I}(1, n)$ and $r_{Q}(1, n)$. The received signal sequence $\{\mathrm{r}(\mathrm{l}, \mathrm{n})\}, \quad 0 \leq n<N M+N_{c p}$, is the transmitted signal sequence $\{\mathrm{s}(1, n)\}$ corrupted by channel noise and the additive white Gaussian noise $\mathrm{w}(\mathrm{l}, \mathrm{n})$. After removing the cyclic prefix, $\{\tilde{r}(l, n)\}$ is split into M OFDM symbols and demodulated using N-point FFT. The recovered complex samples $\hat{X}(l, m, k)$ are decoded to get a pair of numbers, $\left(\hat{d}_{I}, \hat{d}_{Q}\right)$ which represent the maximum likelihood estimates of the quadrature coordinates of the transmitted symbols $\left(d_{I}, d_{Q}\right)$. Thus, the symbols are detected from $\hat{X}(l, m, k)$ based on maximum likelihood (ML) detector and are parallel to serial converted to get the sequence $\{\hat{d}(k)\}$. The binary bit sequence $\{\hat{b}(k)\}$ is recovered from $\{\hat{d}(k)\}$ using a binary mapping. The probability of symbol error is calculated as $\operatorname{Pr}(\hat{d}(k) \neq d(k))$.

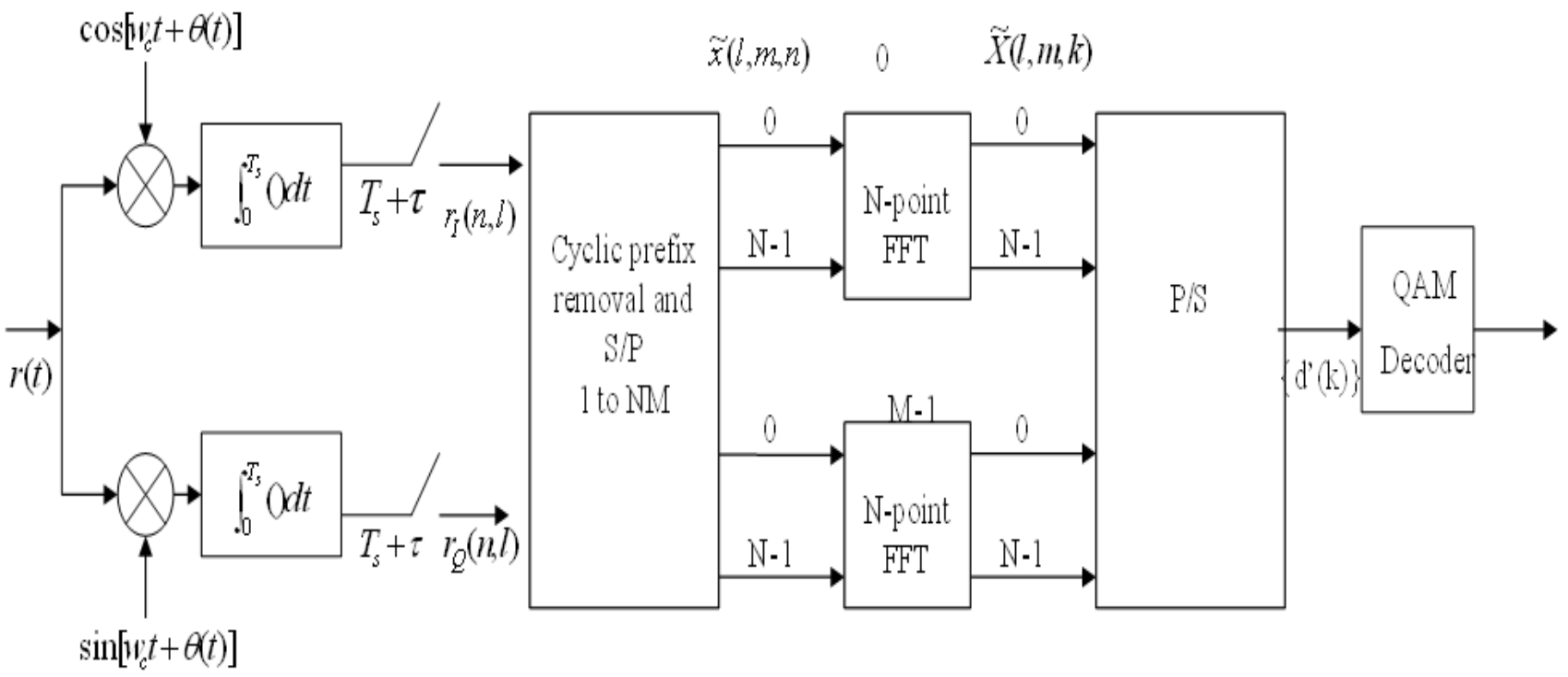

Fig 4. Receiver of MSE-OFDM highlighting the signal processing aspects

We observe that in AWGN channels the OFDM symbols can be separated from a MSE-OFDM frame without any interference. Therefore, the average probability of bit error for the MSE-OFDM system in AWGN channel using MQAM modulation will be same as that for an OFDM system.

In Rayleigh fading channels also, if the channel is slow fading and can be considered to be static over one frame duration, the average probability of bit error for the MSEOFDM system using MQAM modulation will be same as that for an OFDM system. In presence of other interferences in addition to noise, the SNR is replaced by SINR, which is signal-to-interference noise ratio.

We reproduce the results derived for OFDM systems in literature [14-15] for comparison and to derive expressions for MSE-OFDM. The probability of symbol error of OFDM system using MQAM modulation in AWGN is given by [14],

$$
P_{e}=1-\left[1-\frac{2(\sqrt{M}-1)}{\sqrt{M}} Q\left(\sqrt{\frac{3 \gamma_{s}}{M-1}}\right)\right]^{2}
$$

where $\gamma_{s}$ stands for the SNR per symbol under AWGN.

The probability of bit error using QPSK Modulation in AWGN channel is given as [14],

$$
P_{e}=Q\left(\sqrt{2 \gamma_{s}}\right)=Q\left(\sqrt{\frac{2 E_{b}}{N_{o}}}\right)
$$

where $\gamma_{s}$ stands for the SNR per symbol under AWGN, $\mathrm{Eb}$ bhbh is the bit energy, No is the noise variance.

The average symbol error probability of an OFDM system employing MQAM constellation under Rayleigh fading channel can be computed by integrating the 
probability of symbol error under AWGN channel over the PDF of Rayleigh distribution.

The average probability of error under Rayleigh fading can be computed as [15]

$$
P_{e}=\int_{0}^{\infty} P_{e}(\gamma) f_{\gamma}(\gamma) d \gamma
$$

where $f_{\gamma}(\gamma)$ is the PDF of the fading distribution.

The Rayleigh fading distribution is given by [15]

$$
f_{\gamma}(\gamma)=\frac{\gamma}{\sigma^{2}} \exp \left(-\frac{\gamma^{2}}{2 \sigma^{2}}\right), 0 \leq \gamma<\infty=0 \gamma<0
$$

The probability of bit error for the MSE-OFDM system in AWGN channel is found to be same as that of an OFDM system in AWGN and fading channels. Thus we observe that the MSE-OFDM system gives better spectral efficiency without degradation in the performance.

The probability of bit error for the OFDM signal with frequency offset is given as [7]

$$
P_{e}=\int_{-\infty}^{\infty} Q\left(\sqrt{\frac{\gamma_{k}^{2} E_{b}}{\left(\sigma_{I_{k}}^{2}+N_{o}\right)}}\right) f_{\gamma}(\gamma) d \gamma
$$

where $\gamma \mathrm{k}$ is the fading parameter in the $\mathrm{k}$-th subcarrier, $\mathrm{Eb}$ bhbh is the bit energy, No is the noise variance, $f_{\gamma}(\gamma)$ is the joint pdf of $\gamma_{1}, \gamma_{2} \ldots . \gamma_{N}$ and ${\sigma_{I_{k}}}^{2}$ is the variance of ICI given by [7]

$$
\sigma_{I_{k}}^{2}=X^{2} \sum_{\substack{i=0 \\ i \neq k}}^{N-1} \gamma_{i}^{2}\left\{\frac{\sin \pi \Delta k}{N \sin (\pi \Delta k / N)} e^{j \pi \Delta k(N-1) / N}\right\}^{2}
$$

where $\Delta \mathrm{k}$ is the normalized frequency offset defined as ratio of actual frequency offset to subcarrier spacing.

\section{Joint Effects of Carrier Frequency and Timing Offsets}

OFDM systems are very sensitive to frequency and timing errors. We consider the effects of carrier frequency and timing offsets on an MSE-OFDM signal in a multipath fading channel. The probability of bit error for the MSEOFDM signal taking both frequency and timing offset into consideration is derived in this Section. The following assumptions are taken for this analysis:

i Carrier frequency offset $\theta(\mathrm{t})$, uniformly distributed between 0 and $2 \pi$ radians.

ii Timing offset $\tau$, uniformly distributed between 0 and $\mathrm{T}_{\mathrm{b}}$ (one bit duration).

iii Input bits are random, independent and equiprobable,

iv The channel is a ITU-R vehicular-A fading channel.

The received MSE-OFDM signal after demodulation and removing the double frequency term can be written as

$$
r(t)=h(t) s(t-\tau)+I(t)+n(t)
$$

where $s(t)$ is the transmitted signal, $\tau$ is the time delay, $h(t)$ is channel impulse response, $\mathrm{I}(\mathrm{t})$ is inter-carrier interference due to carrier frequency offset and timing offset and $\mathrm{n}(\mathrm{t})$ is Gaussian noise with zero mean and power spectral density No/2.

After removing the cyclic prefix, the whole frame is equalized and split into M OFDM symbols $\hat{x}(m, n)$, each sample of the recovered OFDM symbol can be written as where $\mathrm{H}(\mathrm{k})$ is the channel transfer function at the $\mathrm{k}$-th subcarrier frequency, $\Delta \mathrm{n}$ is the relative timing offset (ratio of the timing offset to the sampling interval), and $\Delta \mathrm{k}$ is the relative frequency offset (ratio between frequency offset to the subcarrier spacing) and $\mathrm{w}(\mathrm{m}, \mathrm{n})$ is the Gaussian noise in $\mathrm{n}$-th sample of $\mathrm{m}$-th OFDM symbol.

$$
\left.\hat{x}(m, n)=\sum_{k=0}^{N-1} X(m, k) H(k) \exp (j 2 \pi(n+m N+\Delta n)(k+\Delta k)) / N\right)+w(m, n), \begin{aligned}
& 0 \leq n<N \\
& 0 \leq m<M
\end{aligned}
$$

These received samples are demodulated using N-point FFT to get samples $\hat{X}(m, k)$

$$
\hat{X}(m, k)=\frac{1}{N} \sum_{n=0}^{N-1} \hat{x}(m, n) \exp (-j 2 \pi n k / N), \quad 0 \leq k<N
$$

substituting $\hat{x}(m, n)$ from eq.(11) in (12), we get

$$
\begin{aligned}
\hat{X}(m, k) & =\frac{1}{N} \sum_{n=0}^{N-1}\left[\sum_{i=0}^{N-1} X(m, i) H(i) \exp (j 2 \pi(n+m N+\Delta n)(i+\Delta k) / N)+w(m, n)\right] \exp (-j 2 \pi n k / N) \\
& =\frac{1}{N} \sum_{n=0}^{N-1} \sum_{i=0}^{N-1} X(m, i) H(i) \exp ((j 2 \pi(n+m N+\Delta n)(i+\Delta k)-n k) / N)+\frac{1}{N} \sum_{n=0}^{N-1} w(m, n) \exp (-j 2 \pi n k / N) \\
& =\frac{1}{N} \sum_{n=0}^{N-1} \sum_{i=0}^{N-1} X(m, i) H(i) \exp ((j 2 \pi(n(i-k+\Delta k)+(m N+\Delta n)(i+\Delta k)) / N)+W(m, k) \\
& =\frac{1}{N} \sum_{n=0}^{N-1} X(m, k) H(k) \exp (j 2 \pi(n \Delta k+(m N+\Delta n)(k+\Delta k)) / N)
\end{aligned}
$$




$$
+\frac{1}{N} \sum_{n=0}^{N-1} \sum_{\substack{i=0 \\
i \neq k}}^{N-1} X(m, i) H(i) \exp \left(j 2 \pi(n(i-k+\Delta k)+(m N+\Delta n)(i+\Delta k) / N)+W(m, k) \quad \begin{array}{l}
0 \leq m<M \\
0 \leq k<N
\end{array}\right.
$$

using the properties of geometric series, we get

$$
\begin{gathered}
\hat{X}(m, k)=X(m, k) H(k) \frac{\sin \pi \Delta k}{N \sin (\pi \Delta k / N)} \exp (j \pi \Delta k(N-1) / N) \exp (j 2 \pi(k+\Delta k) \Delta n / N) \exp (j \pi(M-1) \Delta k) \\
+\sum_{\substack{i=0 \\
i \neq k}}^{N-1} X(m, i) H(i) \frac{\sin \pi(k+\Delta k)}{N \sin (\pi(i-k+\Delta k) / N)} \exp (j \pi(i-k+\Delta k)(N-1) / N) \cdot \exp (j 2 \pi(i+\Delta k)((M-1)+\Delta n) / N)+W(m, k) \quad 0 \leq k<N
\end{gathered}
$$

The fisrt term can be expanded using Taylor series as

$$
X(m, k) H(k) \frac{\sin \pi \Delta k}{N \sin (\pi \Delta k / N)}\left[1+\frac{[\pi \Delta k(N-1)+2 \pi(k+\Delta k) \Delta n+\pi(M-1) \Delta k]}{N}\right]
$$

Substituting (15), eq.(14) can be expressed as

$$
\hat{X}(m, k)=X(m, k) H(k) S(0)+I S I+\sum_{i=0, i \neq k}^{N-1} X(m, i) H(i) S(i-k)+W(m, k) 0 \leq k<N
$$

where $X(m, k)$ denotes the transmitted symbol for the $\mathrm{k}$-th subcarrier, whose amplitude and phase are modified by the frequency offset and timing offset, $\mathrm{S}(\mathrm{k})$ is due to frequency offset given by $(16), W(m, k)$ is the complex Gaussian

$$
X(m, k) H(k) \frac{\sin \pi \Delta k}{\pi \Delta k} \exp (j \pi \Delta k(N-1) / N) \exp (j 2 \pi(k+\Delta k) \Delta n / N) \exp (j \pi(M-1) \Delta k) \quad 0 \leq k<N
$$

The second term in (16) is due to ISI caused by the timing offset and is given by

$$
I S I=X(m, k) H(k)\left[\frac{\pi \Delta k(N-1)+2 \pi(k+\Delta k) \Delta n+\pi(M-1) \Delta k}{N}\right] \quad 0 \leq k<N
$$

The third term in (16) is due to ICI caused by the frequency offset given by

$$
\begin{aligned}
I_{I C I} & \left.=\sum_{\substack{i=0 \\
i \neq k}}^{N-1} X(m, i) H(i) \frac{\sin \pi(k+\Delta k)}{N \sin (\pi(i-k+\Delta k) / N)} \exp (j \pi(i-k+\Delta k)(N-1) / N) \cdot \exp (j 2 \pi(i+\Delta k)((M-1)+\Delta n)) / N\right) \\
& =\sum_{i=0, i \neq k}^{N-1} X(m, i) H(k) S(i-k) \quad 0 \leq k<N
\end{aligned}
$$

The sequence $\mathrm{S}(\mathrm{k})$ depends on carrier frequency offset and timing offset and is given by

$$
S(k)=\frac{\sin \pi(k+\Delta k)}{N \sin \frac{\pi}{N}(k+\Delta k)} \exp \left[j \pi\left(\frac{N-1}{N}\right)(k+\Delta k)((M-1)+\Delta n)\right] 0 \leq k<N
$$

where $\Delta \mathrm{k}$ is the normalized frequency offset and $\Delta \mathrm{n}$ is the relative timing offset.

Since the QAM symbols $\mathrm{X}(\mathrm{m}, \mathrm{i})$ are random variables, the interferences ICI and ISI are also random variables. For large values of $\mathrm{N}$, the power spectral density of ICI and ISI can be approximated by Gaussian process using central limit theorem.

$$
\sigma_{I S I}{ }^{2}=X^{2} H^{2}\left|\frac{\pi \Delta k(N-1)+2 \pi(k+\Delta k) \Delta n+\pi(M-1) \Delta k}{N}\right|^{2}
$$

$$
\begin{aligned}
& \sigma_{I C I}^{2}= \\
& X^{2} \sum_{\substack{i=0 \\
i \neq k}}^{N-1} H_{k}^{2}\left\{\frac{\sin \pi(k+\Delta k)}{N \sin (\pi(i-k+\Delta k) / N)} \exp [j \pi \Delta k(N-1) / N] \exp (j \pi(M-1) \Delta k)\right\}^{2}
\end{aligned}
$$

The probability of bit error for the MSE-OFDM signal taking both frequency and timing offset into consideration is given by

$$
P_{e}=\int_{0}^{\infty} Q\left(\sqrt{\frac{\gamma_{k}^{2} E_{b}}{\left({\left.\sigma_{I C I}^{2}+\sigma_{I S I}^{2}+N_{o}\right)}^{2}\right.}}\right) f(\gamma) d \gamma
$$

where $\gamma_{\mathrm{k}}$ is the fading parameter in the $\mathrm{k}$-th subcarrier given by $\gamma_{k}^{2}=E\left[H(k)^{2}\right], \mathrm{E}_{\mathrm{b}}$ is the bit energy, $\mathrm{N}_{\mathrm{o}}$ is the noise variance, $f_{\gamma}(\gamma)$ is the joint pdf of $\gamma_{1}, \gamma_{2} \ldots . \gamma_{N}, \sigma_{I C I}^{2}$ is the variance of ICI and $\sigma_{I S I}{ }^{2}$ is the variance of ISI given by (21) and (22). 
It is found that the signal amplitude and variance of ICI is multiplied by an extra exponential term in CP-reduced MSE-OFDM signal as compared to OFDM signal. This introduces a phase error due to multiple encapsulated symbols, which can be compensated for by the channel equalizer. The timing offset produces a phase shift in the received signal. The phase shift is more in MSE-OFDM as compared to OFDM systems. The phase error due to multiple encapsulated symbols can be compensated for by the channel equalizer and it does not cause much degradation in the performance.

For FFT-reduced MSE-OFDM system, the IFFT size is reduced keeping the bandwidth constant, so the subcarrier spacing is increased. This reduces the relative frequency offset $\Delta \mathrm{k}$, which is defined as ratio of actual frequency offset to frequency spacing. This reduces the ICI variance and therefore we get better performance in FFT-reduced MSE-OFDM systems as compared to OFDM or CPreduced MSE-OFDM in presence of carrier frequency offset. We have taken relative frequency offset values which are uniformly distributed with zero mean.

\section{Simulation Results}

Table 1. Simulation parameters for MSE-OFDM

\begin{tabular}{lc}
\hline Parameters & Values \\
\hline Number of subcarriers N & 128 \\
$\begin{array}{l}\text { Number of OFDM symbols per } \\
\text { frame M }\end{array}$ & 4 \\
Cyclic prefix $\mathrm{N}_{\mathrm{cp}}$ & 16 \\
Modulation & QPSK \\
Number of pilot carriers $\mathrm{P}$ & 16 \\
Relative frequency offset & Mean $=0.05$, variance $=0.001$ \\
Relative timing offset & Mean $=0.05$, variance $=0.001$ \\
\hline
\end{tabular}

Simulations have been carried out to evaluate the bit error rate (BER) of MSE-OFDM system under different channel conditions. Programming languages $\mathrm{C}++$ and MATLAB have been used for simulation. Stochastic models have been used to generate random numbers for input data. The simulation results are verified using extensive mathematical analysis for each case and the results are found to be close. The small discrepancy in the results is due to the assumptions taken in the analysis. Monte-Carlo simulation is used to get reliable simulation results. The analysis and simulation have been done for QPSK modulation. However, higher QAM modulation can be used to increase the spectral efficiency. The system parameters used are: $\mathrm{N}=128, \mathrm{~B}=4, \mathrm{Ncp}=16$ and modulation scheme is QPSK. The channel is ITU-R defined vehicular A channel. The frequency and timing offsets are assumed to be uniformly distributed random variables with zero mean and variances as mentioned in the simulation results. The relative frequency offset is a uniform random variable with zero mean and variance 0.05 . The simulation parameters are given in Table.1.

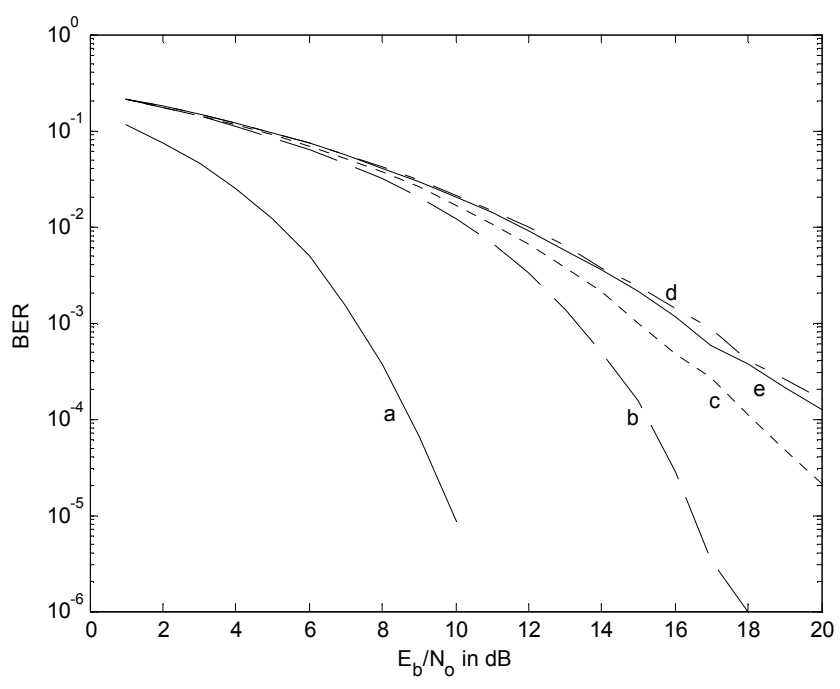

Fig 5. BER for MSE-OFDM system with frequency offset in fading channel (a) BER in AWGN channel (b) BER in fading channel without frequency offset (c) simulation result for BER of FFT-reduced MSE$O F D M$ signal with a relative frequency offset of 0.05 (d) simulation result for BER of CP-reduced MSE-OFDM signal with a relative frequency offset of 0.05 (e) analytical result for CP-reduced system with frequency offset.

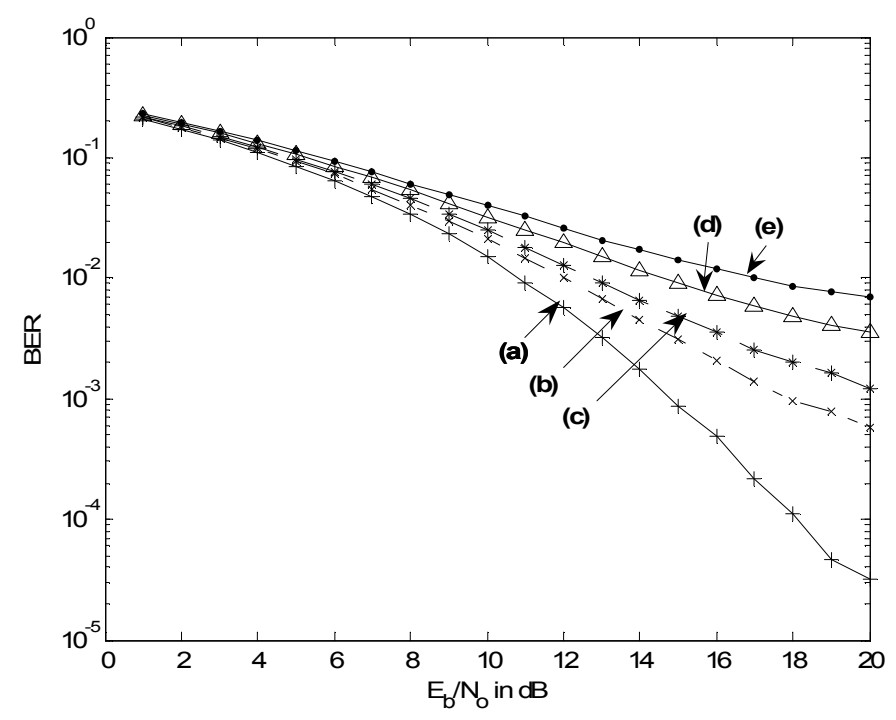

Fig 6. BER for CP-reduced MSE-OFDM system in fading channel for different values of frequency offset (a) without frequency offset (b) with a frequency offset of 0.01 (c) frequency offset of 0.05 (d) frequency offset of 0.08 and (e) frequency offset of 0.1 .

The analytical and simulation results for the probability of bit error for both CP-reduced and FFT-reduced MSEOFDM systems with frequency offset are shown in Fig.5. The BER in AWGN channel is shown in (a) and the BER in multipath fading channel is shown in (b), which are same for both the cases. The simulation result for BER of a 
FFT-reduced MSE-OFDM with a relative frequency offset of 0.05 is shown in (c), the simulation result for BER of a CP-reduced MSE-OFDM with same relative frequency offset of 0.05 is shown in (d) and the analytical result is shown in (e). There is a degradation in BER from 10-5 to $10-4$ at $\mathrm{Eb} / \mathrm{No}$ of $16 \mathrm{~dB}$ due to frequency offset in $\mathrm{CP}$ reduced MSE-OFDM. This degradation is less in case of a FFT-reduced scheme since the number of subcarriers is reduced.

The probability of bit error for the CP-reduced MSEOFDM system with different values of frequency offset in fading is shown in Fig.6. The BER in fading channel without any frequency offset is shown in (a), the BER is found to be $10-4$ at an $\mathrm{Eb} / \mathrm{No}$ of $18 \mathrm{~dB}$. The BER with a uniformly random relative frequency offset with mean 0.01 and variance 0.001 is shown in (b), which is found to be 10-3 at an Eb /No of $18 \mathrm{~dB}$. In (c), the BER with a relative frequency offset of 0.05 is shown and is found to be $2 \times 10$ 3 at the same $\mathrm{Eb} / \mathrm{No}$ of $18 \mathrm{~dB}$. With a relative frequency offset of 0.08, the BER becomes $5 \times 10-3$ at the same $\mathrm{Eb} / \mathrm{No}$ as shown in (d) and with a relative frequency offset of 0.1 , it is 10-2 as shown in (e) for the same value of $\mathrm{Eb} / \mathrm{No}$. Thus, we can see that a small change in frequency offset can degrade the system performance severely.

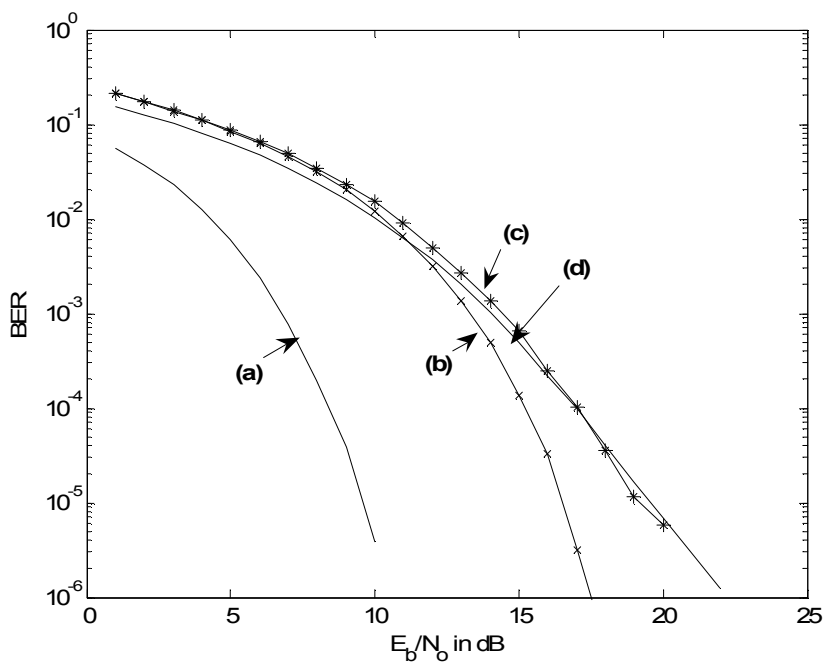

Fig 7. BER for MSE-OFDM system with timing offset in fading channel (a) BER in AWGN channel (b) BER in fading channel without timing offset (c) simulation result for BER in fading channel with a relative timing offset of 0.05 (d) analytical result with timing offset .

The analytical and the simulation results for the probability of bit error for the MSE-OFDM system with timing offset are shown in Fig.7. The BER in AWGN channel is shown in (a), the BER in fading channel is shown in (b), the simulation result for BER with a relative timing offset of 0.05 is shown in (c) and the analytical result for BER with a relative timing offset of 0.05 is shown in (d). There is a degradation in BER from 10-5 to $10-4$ at $\mathrm{Eb} /$ No of $17 \mathrm{~dB}$ due to timing offset.

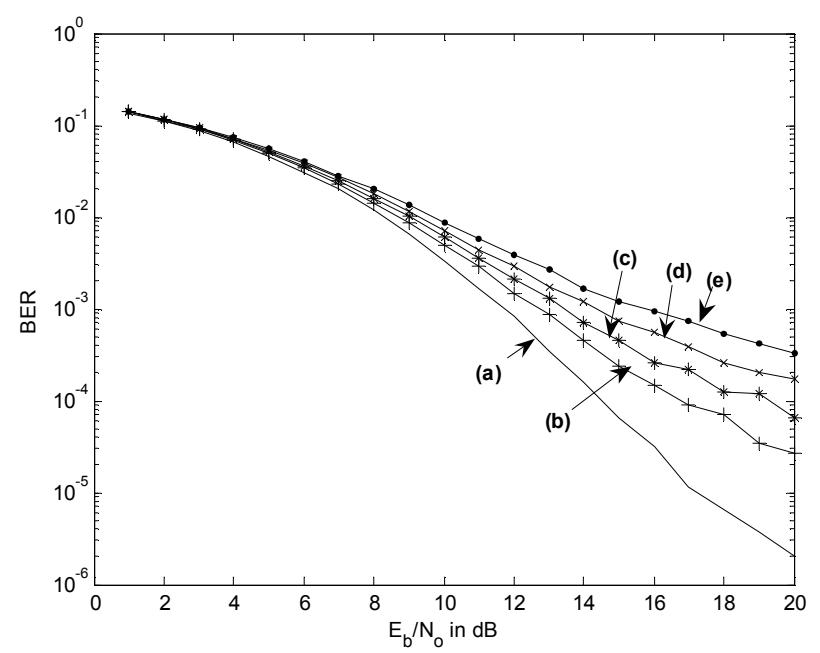

Fig 8. BER for MSE-OFDM system in fading channel for different values of timing offset (a) without timing offset (b) with a timing offset of 0.01 (c) timing offset of 0.05 (d) timing offset of 0.08 and (e) timing offset of 0.1 .

The probability of bit error for the MSE-OFDM system with different values of timing offset in fading is shown in Fig.8. The BER of MSE-OFDM system in fading channel without any timing offset is shown in (a), the BER is found to be $10-5$ at an $\mathrm{Eb} / \mathrm{No}$ of $17 \mathrm{~dB}$. The BER with a uniformly random relative timing offset with mean 0.01 and variance 0.001 is shown in (b), the BER is found to be 10-4 at an $\mathrm{Eb} / \mathrm{No}$ of $17 \mathrm{~dB}$. In (c), the BER with a relative timing offset of 0.05 is shown and is found to be $2 \times 10-4$ at the same $\mathrm{Eb} / \mathrm{No}$ of $17 \mathrm{~dB}$. With a relative timing offset of 0.08 , the BER becomes $5 \times 10-4$ at the same $\mathrm{Eb} / \mathrm{No}$ as shown in (d) and with a relative timing offset of 0.1 , it is 10-3 as shown in (e) for the same value of $\mathrm{Eb} / \mathrm{No}$. It is found that a small change in timing offset can degrade the system performance severely.

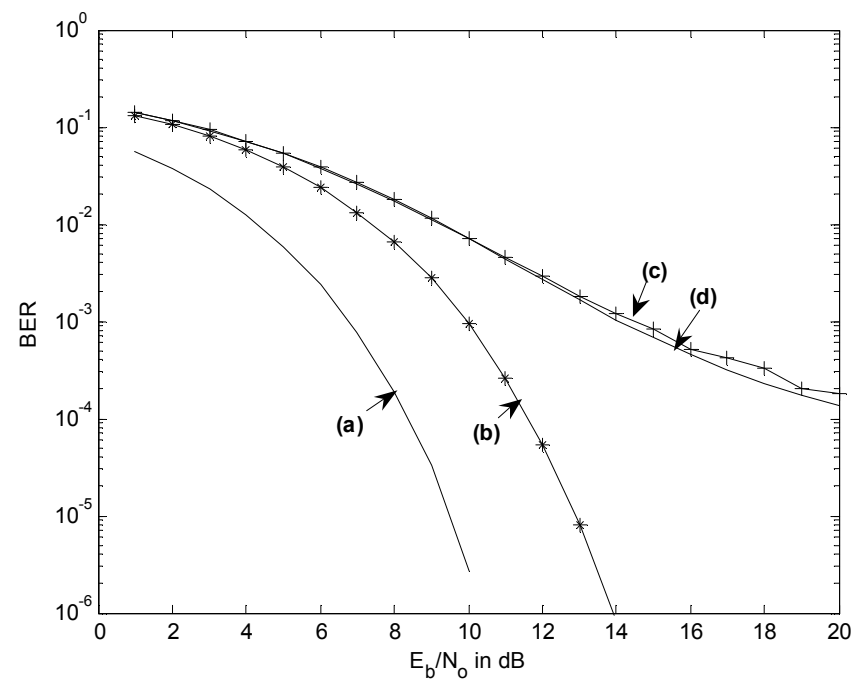

Fig 9. BER for CP-reduced MSE-OFDM system with frequency offset and timing offset in multipath fading channel (a) BER in AWGN channel (b) BER in fading channel without frequency or timing offset (c) simulation result for BER with frequency and timing offset of 0.05 each and (d) analytical result for BER with frequency and timing offset. 
The analytical and the simulation results for the probability of bit error for the CP-reduced MSE-OFDM system with both frequency and timing offset are shown in Fig.9. The BER in AWGN channel is shown in (a), the BER in fading channel is shown in (b), the simulation result for BER with relative frequency offset of 0.05 and relative timing offset of 0.05 is shown in (c) and the analytical result for BER with frequency and timing offset of 0.05 is shown in $(\mathrm{d})$. There is a degradation in BER from 10-6 to $10-3$ at $\mathrm{Eb} / \mathrm{No}$ of $14 \mathrm{~dB}$ due to frequency and timing offsets.

\section{Conclusions}

In this paper, we have analyzed the BER performance of MSE-OFDM systems. MSE-OFDM is very sensitive to synchronization errors. We have analyzed the effects of synchronization errors i.e. carrier frequency offset and timing offset on MSE-OFDM systems and derived expressions for probability of error in presence of these errors, which cause ICI and ISI.

We have done extensive simulations and verified the results analytically. Both analysis and simulation results are presented and are found to be very close. As may be expected, it is observed that the BER performance degrades in presence of synchronization errors. However, this degradation is less in case of FFT-reduced MSEOFDM scheme as compared to conventional OFDM due to reduction in number of subcarriers. The performance improvement by $2-3 \mathrm{~dB}$ has been observed by using this scheme.

\section{References}

[1] S. B. Weinstein and P.M. Ebert, "Data transmission by frequency-division multiplexing using the discrete Fourier transform," IEEE transactions on Communication Technology, vol. 19, pp.628-634, Oct. 1971.

[2] X. Wang, Y. Wu and J. Y. Chouinard, "On the Comparison between Conventional OFDM and MSE-OFDM Systems, "IEEE Global Telecomm. Conference, GLOBECOM'03, pp.35-39, Dec., 2003.

[3] J. Y. Chouinard, X. Wang and Y. Wu, "MSE-OFDM: A New OFDM Transmission Technique with Improved System Performance", International Conference on Accoustics, Speech and Signal Processing, ICASSP 2005, vol.3, 18-23 March,2005.
[4] X. Wang, Y. Wu and J. Y. Chouinard, "System Design and Implementation of Multiple-Symbol Encapsulated OFDM, "IEEE Vehicular Techonology Conference, 2005 VTC, vol.2, 30 May-1 June, 2005.

[5] X. Wang, Y. Wu, J. Y. Chouinard and H. C. Yu, "On the Design and Performance of Multisymbol Encapsulated OFDM Systems," IEEE Transactions on Vehicular Technology, vol. 55, No. 3, May 2006.

[6] E. Sun, K.Yi, B. Tian and X. Wang "A Method for PAPR Reduction in MSE-OFDM Systems,"International Conference on Advanced Information Networking and Applications (AINA'06), vol.2, April, 2006.

[7] M. Morelli, C. C. J. Kuo and M. Pun, "Synchronization Techniques for Orthogonal Frequency Division Multiple Access (OFDMA): A Tutorial Review," Proceedings of the IEEE, vol. 95, No. 7, July 2007.

[8] P.H. Moose, "A technique for orthogonal frequency division multiplexing frequency offset correction," IEEE transactions on communication, vol. 42 , no.10 pp. 29082914, Oct. 2004.

[9] Timothy M. Schmidl and Donald C. Cox, "Robust Frequency and Timing Synchronization for OFDM," IEEE Transactions on Communications, Vol. 45, No. 12, December 1997.

[10] B. Chen, "Maximum likelihood estimation of OFDM carrier frequency offset," IEEE Signal Processing Letters, vol. 9, no.4 pp. 123-126, Apr. 2002.

[11] X. Wang, Y. Wu, H.C.Wu and G. Gagnon, "An MSEOFDM System with Reduced Implementation Complexity Using Pseudo Random Prefix, IEEE Global Telecomm. Conference, GLOBECOM'07, pp.2836-2840, Dec., 2007.

[12] M. Chang and Y. T. Su, "Performance Analysis of Equalized OFDM Systems in Rayleigh Fading", IEEE Transactions on Wireless Communications, vol. 1, No.4, Oct. 2002.

[13] Mishal Al-Gharabally and P. Das, "Performance Analysis of OFDM in Frequency Selective Time-Variant Channels with Application to IEEE 802.16 Broadband Wireless Access", ISSSTA2004, Sydney, Australia, 30 Aug.-2 Sep. 2004.

[14] A Goldsmith, Wireless Communications, Singapore, Cambridge University Press, 2005.

[15] T. S. Rappaport, "Wireless Communications: Principles and Practice," Prentice-Hall, 1996. 